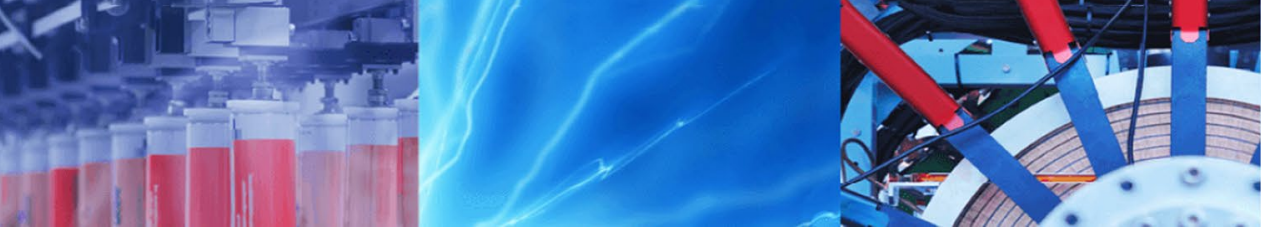

Research Article

\title{
Surprising solutions for some challenging problems arising from boundary layer theory by a new technique: the homotopy contraction mapping technique (HCMT)
}

\author{
Amin Jafarimoghaddam ${ }^{1}$
}

() Springer Nature Switzerland AG 2019

\begin{abstract}
In this work, the homotopy structure $(1-p) L[f]=-p N[f]$ is considered to solve some newly developed nonlinear problems in fluid dynamics. In summary, deformation in homotopy series solutions occurs from the initial trial function to the actual solution. The main challenging part in many similarity equations associated with the boundary layer theory is to identify a certain quantity of the exact solution such as $f^{\prime \prime}(0)$. This quantity is initially unknown and is automatically guessed through the zeroth function. Therefore, it makes sense that in the previous homotopy series solutions one would need at least another parameter i.e. the controlling parameter $(\hbar)$ to handle the convergence through the so-called $\hbar$ -curves analysis and eventually get an approximate value for $f^{\prime \prime}(0)$. Here it is accounted the basic homotopy structure with No controlling parameter $(\hbar)$ and it is shown for the $1^{\text {st }}$ time that the zeroth order solution in homotopy series may be potential to contain some certain quantities of the exact solution, here is to be $f^{\prime \prime}(0)$; i.e. $f_{0}^{\prime \prime}(0)$ could be $f^{\prime \prime}(0)$. This hypothesis is checked through a theorem, being totally linked to the Fixed Point Property (FPP), a topological invariant; i.e. being preserved by any homeomorphism. The theorem enables us Not only to check the validity of the hypothesis, but also to extract the value of the quantity as the unique description of the homotopy series solutions truncated at any order of approximation. The technique is initially introduced in a simple and straightforward manner and then it is applied to some fluid mechanical problems to be further compared with traditional homotopy analysis method, homotopy perturbation method and Numerical solutions. The outcomes indicated an excellent improvement by the present approach as only a few series terms were accounted to obtain highly accurate solutions.
\end{abstract}

Keywords 3-D MHD flow - 2-D flow of UCM fluids · Homotopy analysis method (HAM) · Homotopy perturbation method (HPM) - The homotopy contraction mapping technique (HCMT)

Mathematical Subject Classification Algebraic topology · Fluid dynamics

\section{Introduction}

HAM in individual as described by Liao (e.g. [1-8]) contains several degrees of freedom; the choice for the initial guess (zeroth function, $f_{0}$ ), the auxiliary linear operator (written as: $L[f])$, the controlling parameter $(\hbar)$ and possibly additional homotopy functions. Indeed, as indicated by Liao himself in his numerous published papers (e.g. see [1-8]), these freedoms give the technique an excellent maneuverability to variety of nonlinear problems in a convenient manner. Later on, HPM is systematically described by $\mathrm{He}$ (e.g. [9-15]), in particular fixing the so-called controlling parameter (asserting that such a parameter has been heuristically introduced by Liao) to suggest (as He was

Amin Jafarimoghaddam: Previously at the Department of Aerospace Engineering, K. N. Toosi University of Technology, Tehran, Iran.

Amin Jafarimoghaddam, a.jafarimoghaddam@gmail.com | ${ }^{1}$ Independent Researcher, Tehran, Iran.

SN Applied Sciences (2019) 1:1104 | https://doi.org/10.1007/s42452-019-1114-z

Received: 14 May 2019 / Accepted: 16 August 2019 / Published online: 27 August 2019

SN Applied Sciences

A SPRINGER NATURE journal 
convinced with) a more compact algorithm. P.N. that, as argued by Liao [8], one still needs more rigorous mathematical proofs to distinguish HPM from HAM by the socalled No-secular terms rules; and hence, HPM may not be considered as a more compact algorithm compared to HAM without this proof.

It should be mentioned that the controlling parameter is fixed in HPM and hence, the so-called No-secular condition is somewhat responsible for the convergence of homotopy terms; and without this condition, there would be No control on the convergence and therefore, HPM would become a subset of HAM (see $[8,12]$ to follow the arguments by Liao and He respectively about HPM).

In the present introduction, it is not intended to judge HPM and only the summary of some outcomes of this algorithm is placed to shortly review the literature.

As shown in many references such as [9-21], if the Nosecular term condition is successfully applied, the solution for the nonlinearity can be described in parametric forms (upon having any parameter in the problem) and free from the appearance of controlling parameter which needs further analysis through $\hbar$-curves for the convergence issue.

Reviewing the above cited papers with regard to HPM, authors have mostly sought ways to ease obtaining higher orders of approximation mainly on the basis of parameter expansion methods (as a glance, parameter expansion method is a technique to somewhat manipulate the linear operator with additional parameters; enabling one to remove further secular terms occurring in higher orders of approximation) together with repeating the same rule (the condition of No-secular terms); and in this respect, functionalizing freedoms within the successful and easilyiterative system of HAM has been less sought.

Parameter expansion method has been practiced previously by some authors and it appears that this technique is an advancement in HPM (e.g. see [16-21]); however, in most cases, dealing with complicated systems is the inherent drawback of this technique to obtain higher orders of approximation (e.g. see $[18,21])$. In addition, in some cases, this technique may reveal conditional solution and/ or the solution by this technique may Not be unique and it is Not even clear that the additional solutions are trivial or Not (e.g. see [21]).

By this research, the present author mainly wishes to introduce an open and masked topic in homotopy series analysis.

In homotopy, deformation occurs from the initial guess (zeroth series term) to the actual solution. Suppose that the trial function contains some certain target quantities of the exact solution, here is to be $f^{\prime \prime}(0)$; i.e. $f_{0}^{\prime \prime}(0)$ is supposed to be $f^{\prime \prime}(0)$; and hence, the induction that 'the summation of this quantity generated due to the rest of the series terms should be zero' is immediate. Indeed, this is an extension of the quote, initially postulated by Liao where he defines the initial guess in such a way satisfying the boundary conditions and hence, the boundary conditions for the subsequent linear equations are zero; P.N. that the boundary conditions are the certain quantities of the exact solution (see e.g. [1-8]).

By developing a theorem, it is shown that the above hypothesis is totally linked to the Fixed Point Property (FPP). The theorem Not only assesses the validity of the hypothesis, but also gives the value of the target quantity, being regarded as the unique description of the homotopy series solution at any order of approximation.

In the following, the new approach is systematically defined. Later, the 3-D Magnetohydrodynamics (MHD) flow due to an exponentially stretching surface and the 2-D flow of Upper Convected Maxwell (UCM) fluids over a linearly stretching sheet are solved via various analytic and numeric techniques. Examples illustratively show that the proposed approach is extremely straightforward, highly accurate and promising.

\section{Methodology}

\subsection{The homotopy contraction mapping technique (HCMT)}

The basic idea is to hypothesize that the leading term (series zeroth term) is potential to contain any certain quantity of the exact solution (such as $f^{\prime \prime}(0)$; i.e. $f_{0}^{\prime \prime}(0)$ could be $f^{\prime \prime}(0)$ ). This hypothesis is checked in a detailed manner through a theorem as comes later (denoted as Theorem 2 in this paper).

For this purpose it is initially referred to the following theorem.

Theorem 1 (Banach's Contraction Principle) Let $(X, d)$ represent a complete metric space and $f: X \rightarrow X$ be a contraction, i.e. there exists $k \in(0,1)$ such that for each $x, y \in X: d(f x, f y) \leq k d(x, y)$. Then, $f$ has a unique fixed point in $X$, say $X^{*}$ and for each $x_{0} \in X$ the infinite sequence of function composition converges to this fixed point $\left\{f^{n \rightarrow \infty} x_{0}\right\}=$ fofof $\ldots f x_{0}=x^{*}$ [22]. For more information regarding the proof of the theorem and further extensions interested readers are referred to the in-depth study of Ref. [23-28].

Definitions Consider a nonlinear problem in a general form as: $\mathrm{N}[u(r)]=0, r \in \Omega$ and the boundary conditions as: $B\left(u, \frac{\partial u}{\partial n}\right)=0, r \in \Gamma$. 
Where $N$ is a general differential operator, $u(r)$ is a solution defined in $r \in \Omega, B$ is a boundary condition operator and $\Gamma$ is a boundary of domain $\Omega$.

Consider the homotopy $(1-p) L[u(r), \alpha]=-p N[u(r)]$; with $L$ being an auxiliary linear operator, $p$ the embedding artificial parameter $(p \in[0,1])$ and $\alpha$, an initially unknown parameter. P.N. that $L$ is arbitrary, e.g. $L[(\cdot), \alpha]=\frac{\partial^{n}(\cdot)}{\partial r^{n}}+\alpha \frac{\partial^{m}(\cdot)}{\partial r^{m}}$. In accordance with the above homotopy set-up, the initial solution is linked to $p=0$ and $p=1$ recovers the actual solution and hence, on using the standard expansion $u(r)=u_{0}(r)+p u_{1}(r)+p^{2} u_{2}(r)+\cdots$ the solution is described as $u=\sum_{i=0}^{n \rightarrow \infty} u_{i}$. P.N., as dictated by Liao, the expansion method in HAM is normally Taylor method; however, as suggested by Sajid et al. [29], Taylor method works equivalently as the standard expansion method denoted above.

Lemma Let $\zeta^{*, m}$ be the unique description of a certain quantity of the exact solution (say $\zeta ;$ e.g. $\zeta=f^{\prime \prime}(0)$ ) after the $m$ th order of approximation generated due to the homotopy construction defined above, and $\zeta_{0}^{*}$ the corresponding zeroth guess; i.e. $\sum_{i=0}^{m} \zeta_{i}^{*}=\zeta^{*, m}$; then, $\zeta^{*, m}=\zeta_{0}^{*}$ holds if, $\exists \Lambda=\left\{\zeta_{0} \mid \sum_{i=0}^{m \rightarrow \infty} \zeta_{i}=\zeta\right\}: \zeta \in \Lambda$.

Proof The homotopy series is convergent on $\Lambda$ implying the existence of a unique series solution for the nonlinearity. It follows that there exists a unique description of the solution for the $m$ th order of approximation, i.e. $\zeta^{*, m}$, satisfying $\zeta^{*, m \rightarrow \infty}=\zeta$, and $\zeta^{*, m}$ has No dependency on $\zeta_{0}$. With $\zeta=\zeta^{*, m \rightarrow \infty} \in \Lambda$, we can write $\zeta_{0}=\zeta^{*, m \rightarrow \infty}$; by induction, this also holds for $\zeta^{*, m}$ i.e. $\zeta^{*, m} \in \Lambda$ and we can write $\zeta_{0}=\zeta^{*, m}$. Since $\zeta^{*, m}$ is unique, it follows that $\zeta_{0}$ is also unique, say $\zeta_{0}^{*}$.

Theorem 2 Let $T_{m}\left(\zeta_{0}\right)=\sum_{i=0}^{m} \zeta_{i}$ be a mapping function.

If $T_{m \geq 1}^{n \rightarrow \infty} \zeta_{0}=T_{m} O T_{m} O T_{m} \circ \ldots T_{m}\left(\zeta_{0}\right)=C_{m}$ holds for some $\zeta_{0}$, then $\zeta=C_{m \rightarrow \infty} \in \Lambda$ and $C_{m}=\zeta^{*, m}$ is the non-trivial real solution of $\sum_{i=1}^{m} \zeta_{i}=0$.

Proof Let the condition holds true. Suppose that $T_{m}$ is invertible in a neighborhood of $\zeta^{*, m}$. This gives $T_{m}\left(C_{m}\right)=C_{m}$. Since $C_{m}$ is the unique fixed point of $T_{m}$, then by the lemma $C_{m}=\zeta^{*, m}$ and it is the non-trivial real solution of $\sum_{i=1}^{m} \zeta_{i}=0$. It follows that $\zeta=C_{m \rightarrow \infty} \in \Lambda$.

Remark In fact, for the mth order of approximation by the homotopy construction defined earlier, whatever $\zeta_{0}$ is, it converges to the fixed value $\zeta^{*, m}$ through the recursive pro$\operatorname{cess} \zeta_{0, n+1}=T_{m}\left(\zeta_{0, n}\right), n=0,1,2, \ldots,: \zeta_{0, n \rightarrow \infty}=\zeta^{*, m}=C_{m i}$ meaning that the homotopy structure associated with the linear operator is self-corrector for $\zeta$. Therefore, at this stage it is understandable that the strategy is indeed an insight into HAM preventing from unnecessary extra series terms and providing an optimized solution.

\subsection{Ex. 1: the 3-D MHD flow due to an exponentially stretching surface}

The basic equations and boundary conditions for such a flow can be found in several recent studies such as [30-35]:

$f^{\prime \prime \prime}(\eta)+(f(\eta)+g(\eta)) f^{\prime \prime}(\eta)-2\left(f^{\prime}(\eta)+g^{\prime}(\eta)\right) f^{\prime}(\eta)-M f^{\prime}(\eta)=0$

$g^{\prime \prime \prime}(\eta)+(f(\eta)+g(\eta)) g^{\prime \prime}(\eta)-2\left(f^{\prime}(\eta)+g^{\prime}(\eta)\right) g^{\prime}(\eta)-M g^{\prime}(\eta)=0$

Along with the following boundary conditions:

$f(0)=0, \quad f^{\prime}(0)=1, \quad f^{\prime}(\infty)=0, \quad g(0)=0$,

$g^{\prime}(0)=c, \quad g^{\prime}(\infty)=0$

In Eqs. $1-3, M$ is the magnetic parameter and $c$ is the stretching ratio. Moreover, $c=0$ corresponds to the $2-D$ version, whilst $c=1$ represents the axisymmetric flow.

Unlike the 3-D linearly stretching sheet flow, the above coupled system can be simply broken down (the system is scalable by $g(\eta)=c f(\eta)$ ), a point which seems to be untapped in the literature. It can be easily shown that the above system is convertible to:

$f^{\prime \prime \prime}(\eta)+(c+1) f(\eta) f^{\prime \prime}(\eta)-2(c+1) f^{\prime 2}(\eta)-M f^{\prime}(\eta)=0$

$f(0)=0, \quad f^{\prime}(0)=1, \quad f^{\prime}(\infty)=0$

$g(\eta)=c f(\eta)$

Therefore, it is sufficient to solve Eq. 4. P.N. that the solutions by the above scheme only reveal one possible class of solutions for this nonlinearity; however, it will be compared that these solutions are accurately those already established by other researchers employing numerical analysis and also agree well with the numerical analysis presented in this paper.

First assume $M=0$ :

$f^{\prime \prime \prime}(\eta)+(c+1) f(\eta) f^{\prime \prime}(\eta)-2(c+1) f^{\prime 2}(\eta)=0$

Suppose that $\bar{f}(\eta)$ is the solution for the 2-D version of the above equation, then if the general solution is sought in the form of $A \bar{f}(B \eta)$, one reaches:

$f(\eta)=\frac{1}{\sqrt{c+1}} \bar{f}(\sqrt{c+1} \eta)$

Equation 8 is a consistent solution. Therefore, in the absence of magnetic effect, one should only solve the 2-D version of the flow: 
$\bar{f}^{\prime \prime \prime}(\eta)+\bar{f}(\eta) \bar{f}^{\prime \prime}(\eta)-2 \bar{f}^{\prime 2}(\eta)=0$

$\bar{f}(0)=0, \quad \bar{f}^{\prime}(0)=1, \quad \bar{f}^{\prime}(\infty)=0$

Therefore, for 3-D flow due to an exponentially stretching surface:

$f^{\prime \prime}(0)=\bar{f}^{\prime \prime}(0) \sqrt{c+1}$

$g^{\prime \prime}(0)=\bar{f}^{\prime \prime}(0) c \sqrt{c+1}$

The same fundamental tricks are also applicable to 3-D flow due to a nonlinearly stretching surface (linearly stretching surface is an exception); however, for the sake of the scope of the present study further analysis in this respect is deferred to the future studies.

For $M \neq 0$ Eq. 4, in its original form, is preserved and is to be solved via several methods.

\subsection{Solution by homotopy analysis method (HAM)}

The auxiliary linear operator and the initial guess are chosen as (see e.g. [36-40] to see how HAM may work):

$f_{0}(\eta)=1-e^{-\eta}$

$L(f)=f^{\prime \prime \prime}(\eta)-f^{\prime}(\eta)$

Principally, the problem satisfies:

$(1-p) L\left[\bar{f}(\eta, p)-f_{0}(\eta)\right]=p \hbar N[\bar{f}(\eta, p)]$

with

$\bar{f}(0, p)=0, \quad \bar{f}^{\prime}(0, p)=1, \quad \bar{f}(\infty, p)=0$

In Eq. 15, the nonlinear operator is:

$N[\bar{f}]=\frac{\partial^{3} \bar{f}}{\partial \eta^{3}}+(c+1) \bar{f}(\eta, p) \frac{\partial^{2} \bar{f}}{\partial \eta^{2}}-2(c+1)\left(\frac{\partial \bar{f}}{\partial \eta}\right)^{2}-M \frac{\partial \bar{f}}{\partial \eta}$

In addition, $\hbar$ is the so-called controlling parameter and $p \in[0,1]$ is the homotopy embedding parameter. Clearly:

$\bar{f}(\eta, 0)=f_{0}(\eta), \quad \bar{f}(\eta, 1)=f(\eta)$

When the embedding parameter $p$ deforms from 0 to 1 , the initial guess $f_{0}(\eta)$ approaches $f(\eta)$ (P.N. that this is an artificial topological parameter; and No value between 0 and 1 appears in the HAM calculation process).

Upon using the straightforward expansion technique (Taylor method):

$\bar{f}(\eta, p)=f_{0}(\eta)+\sum_{m=1}^{\infty} f_{m}(\eta) p^{m}$

where

$f_{m}(\eta)=\left.\frac{1}{m !} \frac{\partial^{m} \bar{f}}{\partial p^{m}}\right|_{p=0}$
Since the series converge for $p=1$ :

$f(\eta)=f_{0}(\eta)+\sum_{m=1}^{\infty} f_{m}(\eta)$

For the mth-order deformation:

$L\left[f_{m}(\eta)-\chi_{m} f_{m-1}(\eta)\right]=\hbar \Re_{m}(\eta)$

with the boundary conditions:

$f_{m}(0)=f_{m}^{\prime}(0)=f_{m}^{\prime}(\infty)=0$

where

$\chi_{m}= \begin{cases}0 & m \leq 1 \\ 1 & m>2\end{cases}$

$\begin{aligned} \mathfrak{R}_{m}(\eta)= & f_{m-1}^{\prime}(\eta)+(c+1) \sum_{k=0}^{m-1}\left\{\left(f_{m-1-k}(\eta)\right) f_{k}^{\prime}(\eta)\right\} \\ & -2(c+1) \sum_{k=0}^{m-1}\left\{\left(f_{m-1-k}^{\prime}(\eta)\right) f_{k}^{\prime}(\eta)\right\}-M f_{m-1}^{\prime}(\eta)\end{aligned}$

The above system was solved by developing a symbolic code in MATLAB.

Figure 1 represents the so-called $\hbar$-curves in different order of approximations for the 2-D case $(c=0)$ with $M=0$. The algorithm was truncated at the 15 th order of approximation and it was firmly confirmed $f^{\prime \prime}(0)=-1.2818$ (As a comparison, Liu et al. [30] have reported, employing numerical analysis, that for this case $\left.f^{\prime \prime}(0)=-1.28180856\right)$. Hence, e.g. for $c=0.5$ and $c=1$ we obtain, from Eq. 10 and $11, f^{\prime \prime}(0)=-1.5699$ (Liu et al. [30]: $\left.f^{\prime \prime}(0)=-1.5698884\right), g^{\prime \prime}(0)=-0.7849$ (Liu et al. [30]:

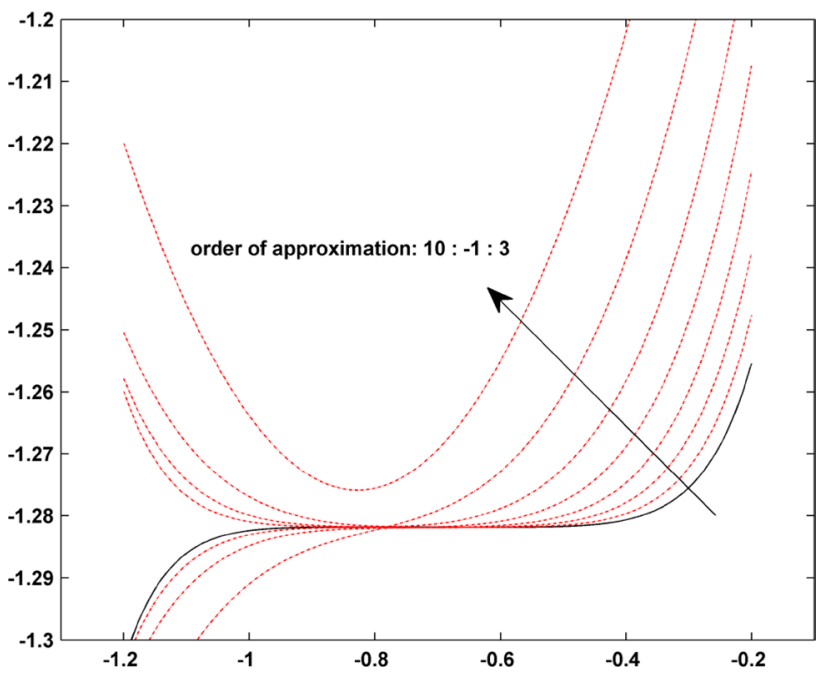

Fig. $1 \hbar$-curves in different orders of approximation for $c=0$, $M=0$ : X-axis and $\mathrm{Y}$-axis are $\hbar$ and $f^{\prime \prime}(0)$ respectively 
$\left.g^{\prime \prime}(0)=-0.78494423\right)$ and $f^{\prime \prime}(0)=-1.8127$ (Liu et al. [30]: $\left.f^{\prime \prime}(0)=-1.8127510\right), g^{\prime \prime}(0)=-1.8127$ (Liu et al. [30]: $\left.g^{\prime \prime}(0)=-1.8127510\right)$ respectively.

Employing $\hbar$-curves analysis for other cases, we could obtain reliable solutions for this problem.

For other cases, having $c \neq 0$ and $M \neq 0$, Table 1 is provided to indicate the behavior of the significant engineering quantity of interest, being secured to the $4^{\text {th }}$ decimal place.

\subsection{Solution by 1st-order homotopy perturbation method (HPM)}

To indicate the basic idea of this method, the following form for a nonlinear equation is considered (see e.g. [41]):

$A(U)-f(r)=0, \quad r \in \Omega$

Boundary conditions are defined as:

$B\left(u, \frac{\partial u}{\partial n}\right)=0, \quad r \in \Gamma$

In above, $A$ is a general function operator, $B$ is a boundary condition operator and $\Gamma$ is a boundary of domain $\Omega$ and $f(r)$ is a known function.

The general operator in Eq. 26 may be decomposed into a linear and a nonlinear operator as:

$L(U)+N(U)-f(r)=0, \quad r \in \Omega$

On using the homotopy $U(r, p): \Omega \times[0,1] \rightarrow R$ satisfying:

$$
\begin{gathered}
H(U, p)=(1-p)\left(L(U)-L\left(U_{0}\right)\right) \\
+p(A(U)-f(r))=0, \\
p \in[0,1], \quad r \in \Omega
\end{gathered}
$$

or

$H(U, p)=\left(L(U)-L\left(U_{0}\right)\right)+p\left(L\left(U_{0}\right)+N(U)-f(r)\right)=0$

In above, $p$ is an embedding artificial parameter (with the same deformation property as dictated in HAM) and $U_{0}$ is an initial approximate solution to Eq. 26. The following properties can be deduced:

$H(U, 0)=\left(L(U)-L\left(U_{0}\right)\right)=0$

$H(U, 1)=(A(U)-f(r))=0$

According to HPM, we normally employ the standard expansion as:

$U=U_{0}+p U_{1}+p^{2} U_{2}+p^{3} U_{3}+p^{4} U_{4}+\cdots$

On choosing $p=1$, the solution of Eq. 26 can be approximated as:
Table $1 f^{\prime \prime}(0)$ by HAM in different cases

\begin{tabular}{llll}
\hline $\mathrm{M}$ & $\mathrm{c}=0$ & $\mathrm{c}=0.5$ & $\mathrm{c}=1$ \\
\hline 0.1 & -1.3211 & -1.6021 & -1.8407 \\
0.2 & -1.3590 & -1.6336 & -1.8682 \\
0.3 & -1.3958 & -1.6644 & -1.8952 \\
0.4 & -1.4316 & -1.6946 & -1.9219 \\
0.5 & -1.4664 & -1.7242 & -1.9481 \\
0.6 & -1.5005 & -1.7533 & -1.9739 \\
0.7 & -1.5337 & -1.7819 & -1.9994 \\
0.8 & -1.5662 & -1.8100 & -2.0246 \\
0.9 & -1.598 & -1.8377 & -2.0494 \\
1 & -1.6292 & -1.8649 & -2.0739 \\
\hline
\end{tabular}

$U=U_{0}+U_{1}+U_{2}+U_{3}+U_{4}+\cdots$

As it can be seen, the HPM is quite similar to HAM with $\hbar=-1$; however, as indicated by He (see e.g. [9-15]), HPM can be distinguished from HAM by e.g. implementing the No-secular term rule as being initially proposed by Lighthill [42], that any term in the series occurring in the solution should be No more singular than the preceding term; however, as discussed in the introduction section, in order to present HPM as a completely distinguished nonlinear solver, one still needs more mathematical proofs.

In the following we apply HPM for the present nonlinear example.

Let us set up the following homotopy equation:

$(1-p) L[f(\eta)]=-p N[f(\eta)]$

The operators are assumed as:

$L[f(\eta)]=f^{\prime \prime \prime}(\eta)-\beta^{2} f^{\prime}(\eta)$

$N[f(\eta)]=f^{\prime \prime \prime}(\eta)+(c+1) f(\eta) f^{\prime \prime}(\eta)-2(c+1) f^{\prime 2}(\eta)-M f^{\prime}(\eta)$

Therefore, one reaches:

$$
\begin{aligned}
& f^{\prime \prime \prime}(\eta)-\beta^{2} f^{\prime}(\eta)+p\left((c+1) f(\eta) f^{\prime \prime}(\eta)-2(c+1) f^{\prime 2}(\eta)\right. \\
& \left.-M f^{\prime}(\eta)+\beta^{2} f^{\prime}(\eta)\right)=0, \quad \beta>0
\end{aligned}
$$

Taking into accounting the standard expansion, $f(\eta)$ is described as:

$f(\eta)=f_{0}(\eta)+f_{1}(\eta) p+f_{2}(\eta) p^{2}+\cdots$

Since, at $p=1$ the original system is recovered, the solution is:

$f(\eta)=f_{0}(\eta)+f_{1}(\eta)+f_{2}(\eta)+\cdots$

On substituting Eq. 39 into Eq. 38 and collecting terms with like powers of $p$, the following zeroth and 1 st-order systems are obtained: 


$$
\begin{aligned}
& p^{0}: f_{0}^{\prime \prime \prime}(\eta)-\beta^{2} f_{0}^{\prime}(\eta)=0 \\
& f_{0}(0)=0, \quad f_{0}^{\prime}(0)=1, \quad f_{0}^{\prime}(\infty)=0 \\
& p^{1}: f_{1}^{\prime \prime \prime}(\eta)-\beta^{2} f_{1}^{\prime}(\eta)+\left((c+1) f_{0}(\eta) f_{0}^{\prime \prime}(\eta)\right. \\
& \left.-2(c+1) f_{0}^{\prime 2}(\eta)-M f_{0}^{\prime}(\eta)+\beta^{2} f_{0}^{\prime}(\eta)\right)=0 \\
& f_{1}(0)=0, \quad f_{1}^{\prime}(0)=0, \quad f_{1}^{\prime}(\infty)=0
\end{aligned}
$$

Solution to Eq. 41 reads:

$f_{0}(\eta)=\frac{1}{\beta}\left(1-e^{-\beta \eta}\right), \quad \beta>0$

Equation 42 gives:

$$
\begin{aligned}
f_{1}(\eta)= & e^{(-\beta \eta)}\left(\frac{3 M+5 c+5}{6 \beta^{3}}-\frac{1}{2 \beta}\right)-e^{(-2 \beta \eta)}\left(\frac{c+1}{6 \beta^{3}}\right) \\
& +\eta e^{(-\beta \eta)}\left(\frac{M+c+1}{2 \beta^{2}}-\frac{1}{2}\right)+\frac{1}{2 \beta}-\frac{2 c+M+2}{3 \beta^{3}}
\end{aligned}
$$

The no-secular terms condition requires:

$\beta=\sqrt{c+M+1}$

Obviously, in the 1st-order HPM, once the secular terms (such as $\eta^{n} e^{(-m \eta)}$ ) are removed, the control is lost on the appearance of these terms in higher orders of approximation. Therefore, it is obtained with ease:

$f(\eta)=f_{0}(\eta)+f_{1}(\eta)$

$f^{\prime \prime}(0)=f_{0}^{\prime \prime}(0)+f_{1}^{\prime}(0)=-\frac{3 M+4 c+4}{3 \sqrt{M+c+1}}$

The results will be compared later.

\subsection{Solution by 2 nd-order homotopy perturbation method (HPM)}

Employing the parameter expansion method (see e.g. [16-21]), the operators in Eq. 35 are defined as:

$L[f(\eta)]=f^{\prime \prime \prime}(\eta)+\left(-\beta_{0}^{2}+p \beta_{1}\right) f^{\prime}(\eta)$

$N[f(\eta)]=f^{\prime \prime \prime}(\eta)+(c+1) f(\eta) f^{\prime \prime}(\eta)-2(c+1) f^{\prime 2}(\eta)-M f^{\prime}(\eta)$

By substitution:

$$
\begin{aligned}
& f^{\prime \prime \prime}(\eta)+\left(-\beta_{0}^{2}+p \beta_{1}\right) f^{\prime}(\eta)+p\left((c+1) f(\eta) f^{\prime \prime}(\eta)\right. \\
& \left.\quad-2(c+1) f^{\prime 2}(\eta)-M f^{\prime}(\eta)-\left(-\beta_{0}^{2}+p \beta_{1}\right) f^{\prime}(\eta)\right)=0
\end{aligned}
$$

The following zeroth, 1 st and 2 nd order systems are then immediate:

$$
\begin{aligned}
& p^{0}: f_{0}^{\prime \prime \prime}(\eta)-\beta_{0}^{2} f_{0}^{\prime}(\eta)=0 \\
& f_{0}(0)=0, \quad f_{0}^{\prime}(0)=1, \quad f_{0}^{\prime}(\infty)=0 \\
& p^{1}: f_{1}^{\prime \prime \prime}(\eta)-\beta_{0}^{2} f_{1}^{\prime}(\eta)+\left((c+1) f_{0}(\eta) f_{0}^{\prime}(\eta)\right. \\
& \left.\quad-2(c+1) f_{0}^{\prime 2}(\eta)-M f_{0}^{\prime}(\eta)+\left(\beta_{0}^{2}+\beta_{1}\right) f_{0}^{\prime}(\eta)\right)=0 \\
& f_{1}(0)=0, \quad f_{1}^{\prime}(0)=0, \quad f_{1}^{\prime}(\infty)=0 \\
& p^{2}: f_{2}^{\prime \prime \prime}(\eta)-\beta_{0}^{2} f_{2}^{\prime}(\eta) \\
& \quad+\left(\begin{array}{ll}
(c+1)\left(f_{0}(\eta) f_{1}^{\prime \prime}(\eta)+f_{1}(\eta) f_{0}^{\prime \prime}(\eta)\right)-2(c+1)\left(2 f_{0}^{\prime}(\eta) f_{1}^{\prime}(\eta)\right)-M f_{1}^{\prime}(\eta) \\
+\left(\beta_{0}^{2}+\beta_{1}\right) f_{1}^{\prime}(\eta)-\beta_{1} f_{0}^{\prime}(\eta)
\end{array}\right)=0 \\
& f_{2}(0)=0, \quad f_{2}^{\prime}(0)=0, \quad f_{2}^{\prime}(\infty)=0
\end{aligned}
$$

Therefore, one reaches:

$f_{0}(\eta)=\frac{1}{\beta_{0}}\left(1-e^{-\beta_{0} \eta}\right), \quad \beta_{0}>0$

$$
\begin{aligned}
f_{1}(\eta)= & e^{\left(-\beta_{0} \eta\right)}\left(\frac{5+5 c+3 M-3 \beta_{1}}{6 \beta_{0}^{3}}-\frac{1}{2 \beta_{0}}\right)-e^{\left(-2 \beta_{0} \eta\right)}\left(\frac{c+1}{6 \beta_{0}^{3}}\right) \\
& +\eta e^{\left(-\beta_{0} \eta\right)}\left(\frac{-1}{2}+\frac{1+c+M-\beta_{1}}{2 \beta_{0}^{2}}\right)+\left(\frac{3 \beta_{1}-3 M-4 c-4}{6 \beta_{0}^{3}}+\frac{1}{2 \beta_{0}}\right)
\end{aligned}
$$

$$
\begin{aligned}
f_{2}(\eta)= & -\eta^{2} e^{\left(-\beta_{0} \eta\right)} \frac{\left(-\beta_{0}^{2}+M-\beta_{1}+c+1\right)^{2}}{8 \beta_{0}^{3}}-e^{\left(-3 \beta_{0} \eta\right)} \frac{(c+1)^{2}}{48 \beta_{0}^{5}} \\
& +e^{\left(-2 \beta_{0} \eta\right)} \frac{(c+1)\left(-9 \beta_{0}^{2}+9 M-9 \beta_{1}+11 c+11\right)}{36 \beta_{0}^{5}} \\
& +\frac{1}{72 \beta_{0}^{5}}\left(K_{1}\right)-\frac{\eta e^{\left(-\beta_{0} \eta\right)}}{24 \beta_{0}^{4}}\left(K_{2}\right)-\frac{e^{\left(-\beta_{0} \eta\right)}}{144 \beta_{0}^{5}}\left(K_{3}\right) \\
& +\eta e^{\left(-2 \beta_{0} \eta\right)} \frac{(c+1)\left(-\beta_{0}^{2}+M-\beta_{1}+c+1\right)}{6 \beta_{0}^{4}} \\
K_{1}= & 27 M^{2}-54 M \beta_{1}-54 M \beta_{0}^{2}+72 M c+72 M+27 \beta_{1}^{2} \\
& +18 \beta_{1} \beta_{0}^{2}-72 \beta_{1} c-72 \beta_{1}+27 \beta_{0}^{4}-72 \beta_{0}^{2} c \\
& -72 \beta_{0}^{2}+52 c^{2}+104 c+52 \\
K_{2}= & 9 M^{2}-18 M \beta_{1}-18 M \beta_{0}^{2}+22 M c+22 M+9 \beta_{1}^{2} \\
& +6 \beta_{1} \beta_{0}^{2}-22 \beta_{1} c-22 \beta_{1}+9 \beta_{0}^{4}-22 \beta_{0}^{2} c \\
& -22 \beta_{0}^{2}+15 c^{2}+30 c+15 \\
K_{3}= & 54 M^{2}-108 M \beta_{1}-108 M \beta_{0}^{2}+180 M c+180 M \\
& +54 \beta_{1}^{2}+36 \beta_{1} \beta_{0}^{2}-180 \beta_{1} c-180 \beta_{1} \\
& +54 \beta_{0}^{4}-180 \beta_{0}^{2} c-180 \beta_{0}^{2}+145 c^{2}+290 c+145
\end{aligned}
$$


Removing the secular terms requires:

$\beta_{0}=\sqrt{\left(\frac{3 K-1}{6}\right)+\sqrt{\left(\frac{3 K-1}{6}\right)^{2}+\frac{\left(-22-44 M+44 K(c+1)-44 M c+36 M K-30 c^{2}-18 K^{2}-60 c-18 M^{2}\right)}{24}}}$

where

$K=c+1+M, \quad \beta_{1}=K-\beta_{0}^{2}$

The approximate solution reads:

$f(\eta)=f_{0}(\eta)+f_{1}(\eta)+f_{2}(\eta)$

Finally, the engineering quantity of interest is obtained as:

$$
\begin{aligned}
f^{\prime \prime}(0) & =f_{0}^{\prime \prime}(0)+f_{1}^{\prime \prime}(0)+f_{2}^{\prime \prime}(0) \\
& =\frac{\left(\begin{array}{c}
9 M^{2}-18 M \beta_{1}-54 M \beta_{0}^{2}+30 M c+30 M+9 \beta_{1}^{2}+18 \beta_{1} \beta_{0}^{2}-30 \beta_{1} c \\
-30 \beta_{1}-27 \beta_{0}^{4}-90 \beta_{0}^{2} c-90 \beta_{0}^{2}+26 c^{2}+52 c+26
\end{array}\right)}{72 \beta_{0}^{3}}
\end{aligned}
$$

\subsection{Solution by the homotopy contraction mapping technique (HCMT)}

Let us take $\zeta=f^{\prime \prime}(0)$. The basic homotopy structure is:

$(1-p) L[f, \alpha]=-p N[f]$

We choose:

$L[f, \alpha]=\frac{\partial^{3} f}{\partial \eta^{3}}-\alpha^{2} \frac{\partial f}{\partial \eta}$

$N[f(\eta)]=f^{\prime \prime \prime}(\eta)+(c+1) f(\eta) f^{\prime \prime}(\eta)-2(c+1) f^{\prime 2}(\eta)-M f^{\prime}(\eta)$

Substituting Eqs. 63 and 62 into Eq. 61 and further employing the standard expansion technique and collecting terms with like power of $p$ (or Taylor method and in HAM) the following systems are reduced:

$p^{0}: f_{0}^{\prime \prime \prime}(\eta)-\alpha^{2} f_{0}^{\prime}(\eta)=0$

$f_{0}(0)=0, \quad f_{0}^{\prime}(0)=1, \quad f_{0}^{\prime}(\infty)=0$ and so on (P.N. that the higher deformation equations are exactly those in the equivalent HAM system).

The technique is to apply the following mapping scheme (see Theorem 2) with

$T_{m}\left(\zeta_{0}\right)=\sum_{i=0}^{m} \zeta_{i}: T_{m \geq 1}^{n \rightarrow \infty} \zeta_{0}=T_{m} \circ T_{m} \circ T_{m} \circ \ldots T_{m}\left(\zeta_{0}\right)=\zeta^{*, m}=C_{m}$

or

$\zeta_{0, n+1}=T_{m}\left(\zeta_{0, n}\right), \quad n=0,1,2, \ldots,: \zeta_{0, n \rightarrow \infty}=\zeta^{*, m}=C_{m}$

$\zeta_{0}\left(=f_{0}^{\prime \prime}(0)\right), \zeta_{1}, \zeta_{2}, \ldots$ are functions of $\alpha$; hence, if there exist some $\zeta_{0}=g(\alpha)$ convergent to a certain value through (67) or (68), the value is regarded as the fixed point of $T_{m}$ and is the unique description of the series solution for $\zeta=f^{\prime \prime}(0)$ at the mth order of truncation; if not, the zeroth term/leading term is Not potential to contain the target quantity $\zeta=f^{\prime \prime}(0)$. In this situation, the linear operator and/or the target quantity should change.

First assume that the series is truncated at the 1st order:

$f_{0}^{\prime \prime}(0)=-\alpha$

$\sum_{i=0}^{1} f_{i}^{\prime \prime}(0)=f_{0}^{\prime \prime}(0)+f_{1}^{\prime \prime}(0)=\chi_{1}\left(f_{0}^{\prime \prime}(0)\right)=\frac{\left(3 f_{0}^{\prime \prime}(0)^{2}+3 M+5 c+5\right)}{6 f_{0}^{\prime \prime}(0)}$

Therefore, the contraction mapping is checked through:

$T_{1}^{n \rightarrow \infty} f_{0}^{\prime \prime}(0)=T_{1} \circ T_{1} \circ T_{1} \circ \ldots T_{1}\left(f_{0}^{\prime \prime}(0)\right)=f_{0}^{\prime \prime *, 1}(0)=C_{1}$

or

$f_{1}(0)=0, \quad f_{1}^{\prime}(0)=0, \quad f_{1}^{\prime}(\infty)=0$

$$
\begin{aligned}
& p^{2}: f_{2}^{\prime \prime \prime}(\eta)-\alpha^{2} f_{2}^{\prime}(\eta)+\left((c+1)\left(f_{1}(\eta) f_{0}^{\prime \prime}(\eta)+f_{0}(\eta) f_{1}^{\prime \prime}(\eta)\right)-2(c+1)\left(2 f_{0}^{\prime}(\eta) f_{1}^{\prime}(\eta)\right)-M f_{1}^{\prime}(\eta)+\alpha^{2} f_{1}^{\prime}(\eta)\right)=0 \\
& f_{2}(0)=0, \quad f_{2}^{\prime}(0)=0, \quad f_{2}^{\prime}(\infty)=0
\end{aligned}
$$




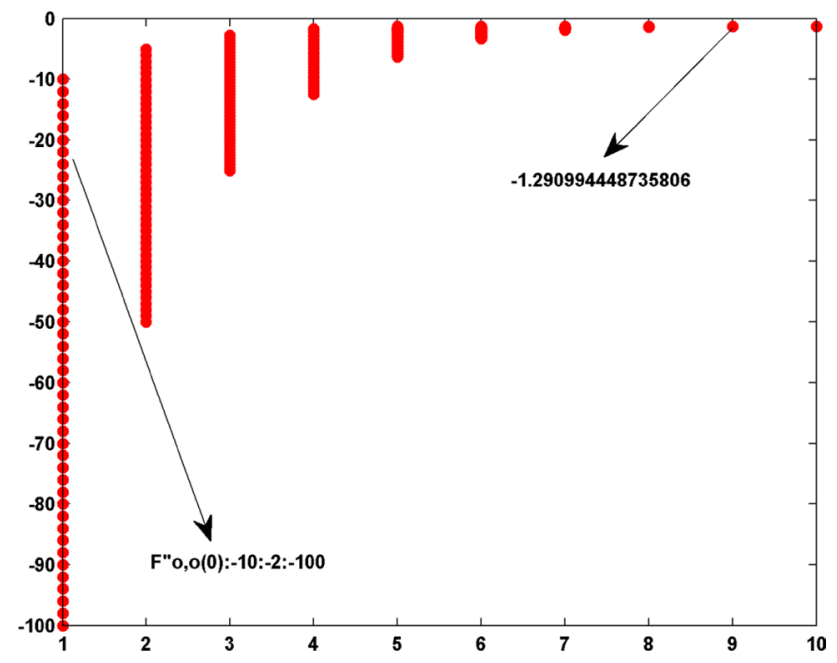

Fig. $2 f_{0, n+1}^{\prime \prime}(0)=T_{1}\left(f_{0, n}^{\prime \prime}(0)\right), n=0,1,2, \ldots,: f_{0, n \rightarrow \infty}^{\prime \prime}(0)=f^{\prime \prime *, 1}(0)=C_{1}$ $\mathrm{X}$-axis is the iteration $(n): c=M=0$

$f_{0, n+1}^{\prime \prime}(0)=T_{1}\left(f_{0, n}^{\prime \prime}(0)\right), \quad n=0,1,2, \ldots,: f_{0, n \rightarrow \infty}^{\prime \prime}(0)=f^{\prime \prime *, 1}(0)=C_{1}$

In this particular example, it can be simply checked that (71) is convergent for any arbitrary choice for $f_{0}^{\prime \prime}(0)$ (see Fig. 2). Moreover, for the 1 st order of truncation, it is possible to establish an analytic relation for $f^{\prime \prime}(0)$ since $\sum_{i=1}^{m} \zeta_{i}=0$ is solvable:

$$
\begin{aligned}
\sum_{i=1}^{1} f_{i}^{\prime \prime}(0) & =f_{1}^{\prime \prime}(0)=\frac{\left(-3 f_{0}^{\prime \prime}(0)^{2}+3 M+5 c+5\right)}{6 f_{0}^{\prime \prime}(0)} \\
& =0 \Rightarrow f_{0}^{\prime \prime}(0)=-\sqrt{\frac{3 M+5 c+5}{3}}
\end{aligned}
$$

Therefore, for the 1st order solution:

$f^{\prime \prime *, 1}(0)=-\sqrt{\frac{3 M+5 c+5}{3}}$

As a comparison, one may check that for the case with $c=M=0 \quad\left(f^{\prime \prime}(0)=-1.2818\right), \quad$ Eq. 74 gives: $f^{\prime \prime}(0)=-\sqrt{\frac{5}{3}} \approx-1.29099$ which is much better than the 1st order HPM (Eq. 47 shows $\left.f^{\prime \prime}(0)=-\frac{4}{3}=-1 . \overline{3}\right)$. Further it is notable that this accuracy is close to the 2 nd order HPM (Eq. 60 gives: $f^{\prime \prime}(0) \approx-1.2752$ ).

In order to increase the accuracy, 5th order of approximation was computed. For this:

$T_{5}^{n \rightarrow \infty} f_{0}^{\prime \prime}(0)=T_{5} \circ T_{5} \circ T_{5} \circ \ldots T_{5}\left(f_{0}^{\prime \prime}(0)\right)=f^{\prime \prime * 5}(0)=C_{5}$

or

$f_{0, n+1}^{\prime \prime}(0)=T_{5}\left(f_{0, n}^{\prime \prime}(0)\right), \quad n=0,1,2, \ldots,: f_{0, n \rightarrow \infty}^{\prime \prime}(0)=f^{\prime \prime *, 5}(0)=C_{5}$

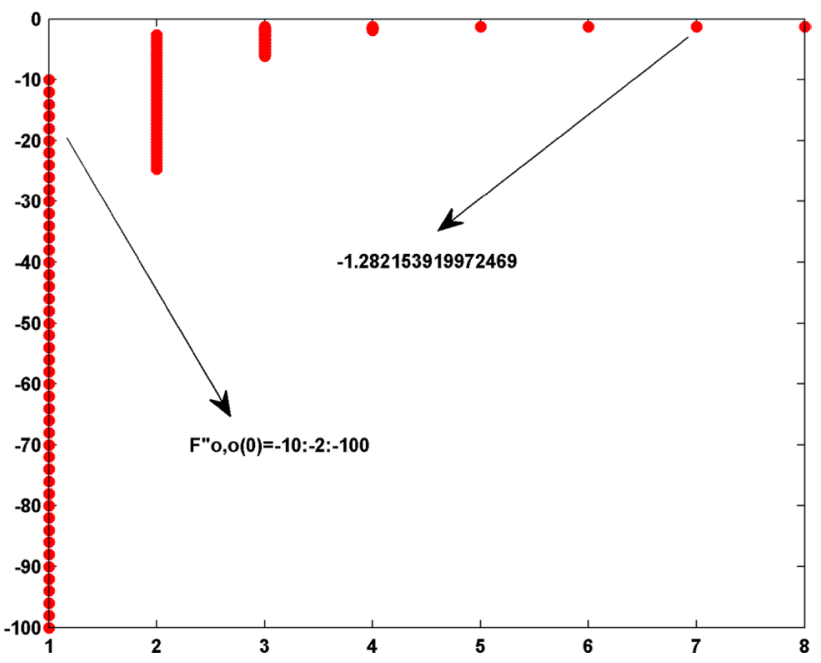

Fig. $3 f_{0, n+1}^{\prime \prime}(0)=T_{5}\left(f_{0, n}^{\prime \prime}(0)\right), n=0,1,2, \ldots,: f_{0, n \rightarrow \infty}^{\prime \prime}(0)=f^{\prime \prime *, 5}(0)=C_{5}$ X-axis is the iteration $(n): c=M=0$

It was computed that:

$T_{5}\left(f_{0}^{\prime \prime}(0)\right)=\overbrace{\frac{-P\left(f_{0}^{\prime \prime}(0)\right)}{108864000 f_{0}^{\prime \prime}(0)^{9}}}^{\sum_{i=1}^{5} f_{i}^{\prime \prime}(0)}+f_{0}^{\prime \prime}(0)$

with

$\begin{aligned} P\left(f_{0}^{\prime \prime}(0)\right)= & A_{0}+A_{1} f_{0}^{\prime \prime}(0)^{2}+A_{2} f_{0}^{\prime \prime}(0)^{4} \\ & +A_{3} f_{0}^{\prime \prime}(0)^{6}+A_{4} f_{0}^{\prime \prime}(0)^{8}+A_{5} f_{0}^{\prime \prime}(0)^{10}\end{aligned}$

$A_{0}=-\left(\begin{array}{c}2976750 M^{5}+24806250 M^{4}(1+c)+85995000 M^{3}(1+c)^{2} \\ +151814250 M^{2}(1+c)^{3}+135329670 M(1+c)^{4}+48539791(1+c)^{5}\end{array}\right)$

$A_{1}=\left(\begin{array}{c}173995290(1+c)^{4}+19136250 M^{4}+127575000 M^{3}(1+c) \\ +331695000 M^{2}(1+c)^{2}+390379500 M(1+c)^{3}\end{array}\right)$

$A_{2}=-\left(\begin{array}{c}53581500 M^{3}+267907500 M^{2}(1+c)+464373000 M(1+c)^{2} \\ +273265650(1+c)^{3}\end{array}\right)$

$A_{3}=\left(89302500 M^{2}+297675000 M(1+c)+257985000(1+c)^{2}\right)$

$A_{4}=-(133953750 M+223256250(1+c))$

$A_{5}=(-26790750+108864000)$

The fixed point is one of the real polynomial roots $\left(\sum_{i=1}^{5} f_{i}^{\prime \prime}(0)=0 \Rightarrow P\left(f_{0}^{\prime \prime}(0)\right)=0\right)$ which certainly shows itself through the recursive process (see Fig. 3). By analysis, 5 th order of approximation in each certain values of $c$ 
and $M$ gives solutions in which the maximum deviation, compared to the high-order HAM results, Does Not exceed $0.03 \%$.

\subsection{A perturbation solution valid for large $M$}

Introducing the new variable $\xi=\sqrt{M} \eta$ Eq. 4 and the associated boundary conditions become:

$f^{\prime \prime \prime}(\xi)-f^{\prime}(\xi)+\varepsilon\left((c+1) f(\xi) f^{\prime \prime}(\xi)-2(c+1) f^{\prime 2}(\xi)\right)=0$

$f(0)=0, \quad f^{\prime}(0)=\frac{1}{\sqrt{M}}, \quad f^{\prime}(\infty)=0$

Let us choose:

$\varepsilon=\frac{1}{\sqrt{M}}: M \rightarrow \infty \Rightarrow \varepsilon \rightarrow 0$

Employing the standard expansion $f(\xi)=f_{0}(\xi)+\varepsilon f_{1}(\xi)+\varepsilon^{2} f_{2}(\xi)+\cdots$ the following zeroth and 1 st order systems can be obtained:

$f_{0}^{\prime \prime \prime}(\xi)-f_{0}^{\prime}(\xi)=0$

$f_{0}(0)=0, \quad f_{0}^{\prime}(0)=\frac{1}{\sqrt{M}}, \quad f_{0}^{\prime}(\infty)=0$

$f_{1}^{\prime \prime \prime}(\xi)-f_{1}^{\prime}(\xi)+\left((c+1) f_{0}(\xi) f_{0}^{\prime \prime}(\xi)-2(c+1) f_{0}^{\prime 2}(\xi)\right)=0$

$f_{1}(0)=0, \quad f_{1}^{\prime}(0)=0, \quad f_{1}^{\prime}(\infty)=0$ $\alpha$ is initially missing; hence, a shooting procedure is required to treat the problem as an IVP. Besides, one of the basic challenges to obtain much more accurate results is to deal with the boundary condition as $\eta \rightarrow \infty$, namely, $\eta_{\infty}$ (usually a trial and error procedure is followed to handle this situation). In fact, changing the involved parameters will affect the boundary layer thickness; i.e. the real value of $\left|F^{\prime}\left(\eta_{\infty}\right)\right|$ in a fixed $\eta_{\infty}$ (e.g. $\left.\eta_{\infty}=10\right)$ is highly dependent on $c$ and $M$, expressing rather drastic behaviors. Therefore, in order to retain the same representation of the real solution at the infinity while changing the engaged parameters and searching for a suitable zeroth correspondence $(\alpha), \eta_{\infty}$ (with a fixed error tolerance) or the error tolerance (with a fixed $\eta_{\infty}$ ) should also change. Here, it is suggested using the 1st-order HPM results and following the former scenario to handle the uncertainties. In the present study, on using Eqs. 43-45, the following scheme was considered with $\left|F^{\prime}\left(\eta_{\infty}\right)\right|=10^{-6}$.

$\eta_{\infty}=\frac{1}{(1+c+M)} \operatorname{Ln}\left[\frac{(2+2 c+3 M)}{3(1+c+M)\left(\left|F^{\prime}\left(\eta_{\infty}\right)\right|\right)}\right]$

5th order Runge-Kutta was employed for discretization purpose. An iterative shooting procedure (to the 5 th decimal place) was then pursued.

Table 2 shows a comparison between the methods.

\subsection{Ex. 2: The 2-D flow of UCM fluid over a linearly stretching sheet}

The basic equations for such a flow can be found in several studies e.g. [43-48]:

By solution:

$f(\xi) \approx f_{0}(\xi)+\varepsilon f_{1}(\xi)=\frac{1}{\sqrt{M}}\left(\left(1-e^{-\xi}\right)+\frac{5(c+1)}{6 M} e^{-\xi}-\frac{2(c+1)}{3 M}+\frac{(c+1)}{2 M} \xi e^{-\xi}-\frac{(c+1)}{6 M} e^{-2 \xi}\right)$

Retrieving the original variable it is obtained with ease:

$f^{\prime \prime}(0)=-\sqrt{M}-\frac{5(c+1)}{6 \sqrt{M}}$

\subsection{Numerical solutions}

Equation 4 is initially considered in the following form:

$$
\begin{aligned}
& F=f(\eta) \\
& F^{\prime}=Y \\
& Y^{\prime}=Z \\
& Z^{\prime}+(c+1) F Z-2(c+1) Y^{2}-M Y=0 \\
& F(0)=0, \quad Y(0)=1, \quad Z(0)=\alpha
\end{aligned}
$$

$$
f^{\prime \prime \prime}(\eta)+f(\eta) f^{\prime \prime}(\eta)-f^{\prime}(\eta)^{2}+\beta\left(2 f(\eta) f^{\prime}(\eta) f^{\prime \prime}(\eta)-f(\eta)^{2} f^{\prime \prime \prime}(\eta)\right)=0
$$

The associated boundary conditions are:

$f(0)=0, \quad f^{\prime}(0)=1, \quad f^{\prime}(\infty)=0$

In above, $\beta$ is Deborah number.

\subsection{Solution by homotopy analysis method (HAM)}

The auxiliary linear operator and the initial guess are chosen as before:

$$
\begin{aligned}
& f_{0}(\eta)=1-e^{-\eta} \\
& L(f)=f^{\prime \prime \prime}(\eta)-f^{\prime}(\eta)
\end{aligned}
$$


Table 2 Comparison of the applied methods for $-f^{\prime \prime}(0)$ where $c=0.5$

\begin{tabular}{lllllll}
\hline M & R-K/5th order & HAM & HCMT/5th & HCMT/1st & HPM/1st & HPM/2nd \\
\hline 0.1 & 1.60209 & 1.6021 & 1.6024 & 1.6125 & 1.6602 & 1.6118 \\
0.2 & 1.63357 & 1.6336 & 1.6338 & 1.6432 & 1.6873 & 1.6448 \\
0.3 & 1.66439 & 1.6644 & 1.6646 & 1.6733 & 1.7143 & 1.6765 \\
0.4 & 1.69459 & 1.6946 & 1.6947 & 1.7029 & 1.7411 & 1.7072 \\
0.5 & 1.72423 & 1.7242 & 1.7243 & 1.7321 & 1.7678 & 1.7371 \\
0.6 & 1.75333 & 1.7533 & 1.7534 & 1.7607 & 1.7942 & 1.7663 \\
0.7 & 1.78192 & 1.7819 & 1.7819 & 1.7889 & 1.8203 & 1.7948 \\
0.8 & 1.81002 & 1.8100 & 1.8100 & 1.8166 & 1.8463 & 1.8228 \\
0.9 & 1.83769 & 1.8377 & 1.8377 & 1.8439 & 1.8719 & 1.8503 \\
1 & 1.86493 & 1.8649 & 1.8649 & 1.8708 & 1.8974 & 1.8773 \\
\hline
\end{tabular}

Following Liao (see e.g. [1-8]), the homotopy is constructed as:

$(1-p) L\left[\bar{f}(\eta, p)-f_{0}(\eta)\right]=p \hbar N[\bar{f}(\eta, p)]$

with

$\bar{f}(0, p)=0, \quad \bar{f}^{\prime}(0, p)=1, \quad \bar{f}(\infty, p)=0$

In above, the nonlinear operator is:

$$
\begin{aligned}
N[\bar{f}(\eta, p)]= & \frac{\partial^{3} \bar{f}}{\partial \eta^{3}}+\bar{f}(\eta, p) \frac{\partial^{2} \bar{f}}{\partial \eta^{2}}-\left(\frac{\partial \bar{f}}{\partial \eta}\right)^{2} \\
& +\beta\left(2 \bar{f}(\eta, p)\left(\frac{\partial \bar{f}}{\partial \eta}\right) \frac{\partial^{2} \bar{f}}{\partial \eta^{2}}-\bar{f}(\eta, p)^{2} \frac{\partial^{3} \bar{f}}{\partial \eta^{3}}\right)
\end{aligned}
$$

Following the same procedure, as in the previous problem, for the mth-order deformation one obtains:

$L\left[f_{m}(\eta)-\chi_{m} f_{m-1}(\eta)\right]=\hbar \Re_{m}(\eta)$

With the boundary conditions:

$f_{m}(0)=f_{m}^{\prime}(0)=f_{m}^{\prime}(\infty)=0$

where

$$
\begin{aligned}
& \chi_{m}= \begin{cases}0 & m \leq 1 \\
1 & m>2\end{cases} \\
& \mathfrak{R}_{m}(\eta)= f_{m-1}^{\prime \prime \prime}(\eta)+\sum_{k=0}^{m-1}\left\{\left(f_{m-1-k}(\eta)\right) f_{k}^{\prime}(\eta)\right\}-\sum_{k=0}^{m-1}\left\{\left(f_{m-1-k}^{\prime}(\eta)\right) f_{k}^{\prime}(\eta)\right\} \\
&+\beta \sum_{k=0}^{m-1}\left\{\left(f_{m-1-k}(\eta)\right) \sum_{l=0}^{k}\left\{2 f_{k-1}^{\prime}(\eta) f_{l}^{\prime \prime}(\eta)-f_{k-l}(\eta) f_{l}^{\prime \prime}(\eta)\right\}\right\}
\end{aligned}
$$

Figures 4 and 5 show the behavior of the so-called $\hbar$ -curves for 2 different stages. In addition, Table 3 provides the behavior of this significant quantity of interest in various stages.

\subsection{Solution by 1 st-order homotopy perturbation method (HPM)}

Let us consider the following classic homotopy transformation:

$$
\begin{aligned}
& f^{\prime \prime \prime}(\eta)-k^{2} f^{\prime}(\eta)+p\left(f(\eta) f^{\prime \prime}(\eta)-f^{\prime}(\eta)^{2}\right. \\
& \quad+\beta\left(2 f(\eta) f^{\prime}(\eta) f^{\prime \prime}(\eta)-f(\eta)^{2} f^{\prime \prime \prime}(\eta)\right) \\
& \left.\quad+k^{2} f^{\prime}(\eta)\right)=0, \quad k>0
\end{aligned}
$$

With the standard expansion:

$f(\eta)=f_{0}(\eta)+p f_{1}(\eta)+p^{2} f_{2}(\eta)+\cdots$

The following zeroth and 1 st order systems are then immediate:

$f_{0}^{\prime \prime \prime}(\eta)-k^{2} f_{0}^{\prime}(\eta)=0$

$f_{0}(0)=0, \quad f_{0}^{\prime}(0)=1, \quad f^{\prime}(\infty)=0$

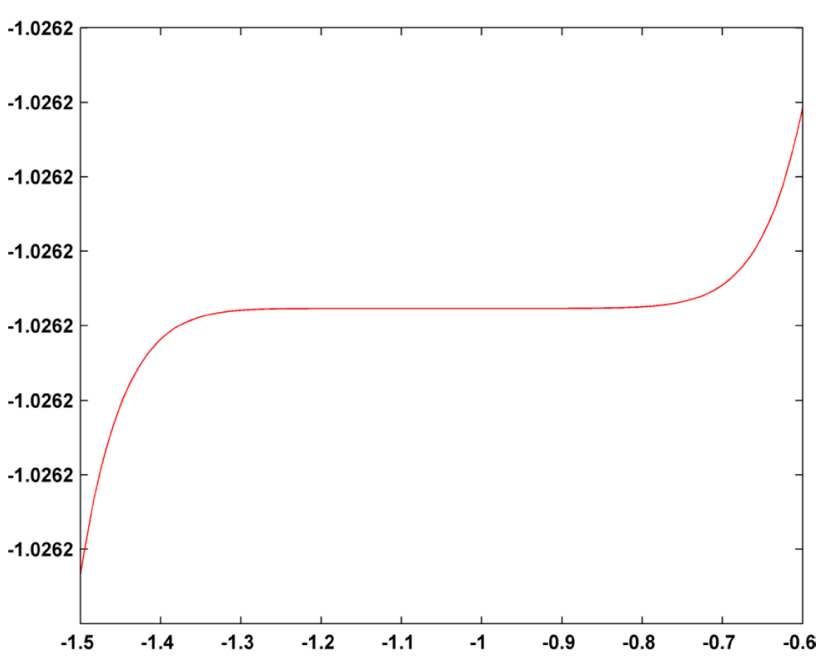

Fig. $4 \hbar$-curve for $\beta=1 / 10$ in 10th order of approximation: $\mathrm{X}$-axis and $Y$-axis are $\hbar$ and $f^{\prime \prime}(0)$ respectively 


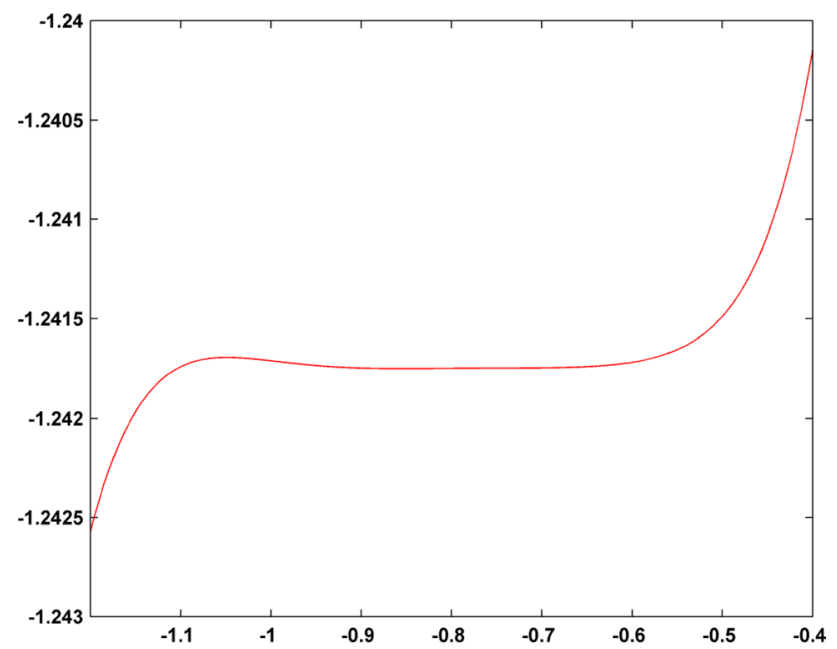

Fig. $5 \hbar$-curve for $\beta=1$ in 10th order of approximation: $\mathrm{X}$-axis and $\mathrm{Y}$-axis are $\hbar$ and $f^{\prime \prime}(0)$ respectively

$$
\begin{aligned}
& f_{1}^{\prime \prime \prime}(\eta)-k^{2} f_{1}^{\prime}(\eta)+f_{0}(\eta) f_{0}^{\prime \prime}(\eta)-f_{0}^{\prime}(\eta)^{2} \\
& \quad+\beta\left(2 f_{0}(\eta) f_{0}^{\prime}(\eta) f_{0}^{\prime \prime}(\eta)-f_{0}(\eta)^{2} f_{0}^{\prime \prime \prime}(\eta)\right)+k^{2} f_{0}^{\prime}(\eta)=0 \\
& f_{1}(0)=f_{1}^{\prime}(0)=f_{1}^{\prime \prime}(\infty)=0
\end{aligned}
$$

Upon solving the above systems and removing the secular terms it is eventually obtained with ease:

$f^{\prime \prime}(0)=f_{0}^{\prime \prime}(0)+f_{1}^{\prime \prime}(0)=-\frac{4+3 \beta}{4 \sqrt{1+\beta}}$

\subsection{Solution by HCMT}

Consider the basic homotopy structure $(1-p) L[f, \alpha]=-p N[f]$ with $L[f, \alpha]=\frac{\partial^{3} f}{\partial \eta^{3}}-\alpha^{2} \frac{\partial f}{\partial \eta}$ and $N[f]=f^{\prime \prime \prime}+f f^{\prime \prime}-f^{\prime 2}+\beta\left(2 f f^{\prime} f^{\prime \prime}-f^{2} f^{\prime \prime \prime}\right)$.

Employing the standard expansion $f=f_{0}+p f_{1}+p^{2} f_{2}+\cdots$ it is obtained:

$f_{0}^{\prime \prime \prime}(\eta)-\alpha^{2} f_{0}^{\prime}(\eta)=0$

$f_{0}(0)=0, \quad f_{0}^{\prime}(0)=1, \quad f^{\prime}(\infty)=0$

$$
\begin{aligned}
& f_{1}^{\prime \prime \prime}(\eta)-\alpha^{2} f_{1}^{\prime}(\eta)+f_{0}(\eta) f_{0}^{\prime \prime}(\eta)-f_{0}^{\prime}(\eta)^{2} \\
& \quad+\beta\left(2 f_{0}(\eta) f_{0}^{\prime}(\eta) f_{0}^{\prime \prime}(\eta)-f_{0}(\eta)^{2} f_{0}^{\prime \prime}(\eta)\right)+\alpha^{2} f_{0}^{\prime}(\eta)=0 \\
& f_{1}(0)=f_{1}^{\prime}(0)=f_{1}^{\prime}(\infty)=0
\end{aligned}
$$

and so on.

The contraction mapping is checked through:

$$
T_{m \geq 1}^{n \rightarrow \infty} \zeta_{0}=T_{m} \circ T_{m} \circ T_{m} \circ \ldots T_{m}\left(\zeta_{0}\right)=\zeta^{*, m}=C_{m}
$$

or:

$\zeta_{0, n+1}=T_{m}\left(\zeta_{0, n}\right), \quad n=0,1,2, \ldots,: \zeta_{0, n \rightarrow \infty}=\zeta^{*, m}=C_{m}$

Let us choose $\zeta=f^{\prime \prime}(0)$ and the 1st order approximation reads:

$f_{0}^{\prime \prime}(0)=-\alpha$

$$
\sum_{i=0}^{1} f_{i}^{\prime \prime}(0)=f_{0}^{\prime \prime}(0)+f_{1}^{\prime \prime}(0)=T_{1}\left(f_{0}^{\prime \prime}(0)\right)=\frac{\left(2 f_{0}^{\prime \prime}(0)^{2}+\beta+2\right)}{4 f_{0}^{\prime \prime}(0)}
$$

Therefore:

$T_{1}^{n \rightarrow \infty} f_{0}^{\prime \prime}(0)=T_{1} \circ T_{1} \circ T_{1} \circ \ldots T_{1}\left(f_{0}^{\prime \prime}(0)\right)=f^{\prime \prime *, 1}(0)=C_{1}$

or

$$
f_{0, n+1}^{\prime \prime}(0)=T_{1}\left(f_{0, n}^{\prime \prime}(0)\right), \quad n=0,1,2, \ldots,: f_{0, n \rightarrow \infty}^{\prime \prime}(0)=f^{\prime \prime *, 1}(0)=C_{1}
$$

Figure 6 shows the contraction mapping behavior.

By Theorem 2, since the 1st order system is solvable:

$$
\begin{aligned}
\sum_{i=1}^{1} f_{i}^{\prime \prime}(0)=f_{1}^{\prime \prime}(0) & =\frac{\left(-2 f_{0}^{\prime \prime}(0)^{2}+\beta+2\right)}{4 f_{0}^{\prime \prime}(0)} \\
& =0 \Rightarrow f_{0}^{\prime \prime}(0)=f^{\prime \prime *, 1}(0)=-\sqrt{\frac{\beta+2}{2}}
\end{aligned}
$$

In order to increase the accuracy, 4th order of approximation was computed. For this:

$T_{4}^{n \rightarrow \infty} f_{0}^{\prime \prime}(0)=T_{4} O T_{4} O T_{4} O \ldots T_{4}\left(f_{0}^{\prime \prime}(0)\right)=f^{\prime \prime *, 4}(0)=C_{4}$

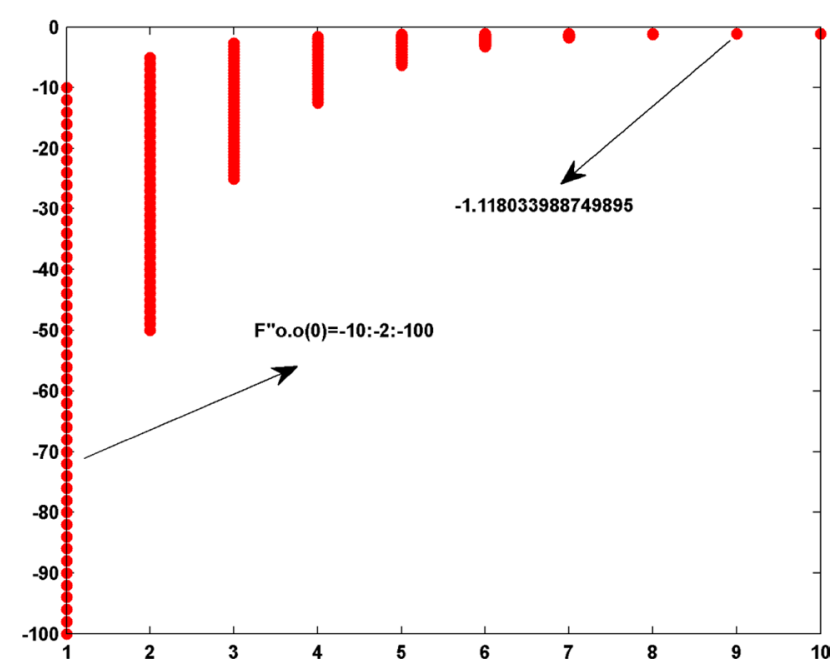

Fig. $6 f_{0, n+1}^{\prime \prime}(0)=T_{1}\left(f_{0, n}^{\prime \prime}(0)\right), n=0,1,2, \ldots,: f_{0, n \rightarrow \infty}^{\prime \prime}(0)=f^{\prime \prime *, 1}(0)=C_{1}$ $\mathrm{X}$-axis is the iteration $(n): \beta=1 / 2$ 
Table $3 f^{\prime \prime}(0)$ in different stages, secured to the 4th decimal place after 15th-order of approximation

\begin{tabular}{llllll}
\hline$\beta$ & $f^{\prime \prime}(0)$ & $\beta$ & $f^{\prime \prime}(0)$ & $\beta$ & $f^{\prime \prime}(0)$ \\
\hline 0.1 & -1.0262 & 0.6 & -1.1501 & 1.1 & -1.2637 \\
0.2 & -1.0519 & 0.7 & -1.1736 & 1.2 & -1.2854 \\
0.3 & -1.0771 & 0.8 & -1.1967 & 1.3 & -1.3067 \\
0.4 & -1.1019 & 0.9 & -1.2194 & 1.4 & -1.3277 \\
0.5 & -1.1262 & 1 & -1.2417 & 1.5 & -1.3484 \\
\hline
\end{tabular}

Table 4 Comparison of the applied methods for $-f^{\prime \prime}(0)$

\begin{tabular}{llllll}
\hline$\beta$ & R-K/5th order & HAM & HCMT/4th & HCMT/1st & HPM \\
\hline 0.1 & 1.02617 & 1.0262 & 1.0262 & 1.0247 & 1.0250 \\
0.2 & 1.05186 & 1.0519 & 1.0519 & 1.0488 & 1.0498 \\
0.3 & 1.07708 & 1.0771 & 1.0771 & 1.0724 & 1.0744 \\
0.4 & 1.10186 & 1.1019 & 1.1019 & 1.0954 & 1.0987 \\
0.5 & 1.12619 & 1.1262 & 1.1262 & 1.1180 & 1.1227 \\
0.6 & 1.15008 & 1.1501 & 1.1502 & 1.1402 & 1.1463 \\
0.7 & 1.17357 & 1.1736 & 1.1737 & 1.1619 & 1.1696 \\
0.8 & 1.19665 & 1.1967 & 1.1968 & 1.1832 & 1.1926 \\
0.9 & 1.21935 & 1.2194 & 1.2195 & 1.2041 & 1.2152 \\
1 & 1.24168 & 1.2417 & 1.2419 & 1.2247 & 1.2374 \\
\hline
\end{tabular}

\section{or}

$f_{0, n+1}^{\prime \prime}(0)=T_{4}\left(f_{0, n}^{\prime \prime}(0)\right), \quad n=0,1,2, \ldots,: f_{0, n \rightarrow \infty}^{\prime \prime}(0)=f^{\prime \prime *, 4}(0)=C_{4}$

It was computed that:

$T_{4}\left(f_{0}^{\prime \prime}(0)\right)=\overbrace{\frac{-P\left(f_{0}^{\prime \prime}(0)\right)}{1016064000 f_{0}^{\prime \prime}(0)^{7}}}^{\sum_{i=1}^{4} f_{i}^{\prime \prime}(0)}+f_{0}^{\prime \prime}(0)$

with

$P\left(f_{0}^{\prime \prime}(0)\right)=A_{0}+A_{1} f_{0}^{\prime \prime}(0)^{2}+A_{2} f_{0}^{\prime \prime}(0)^{4}+A_{3} f_{0}^{\prime \prime}(0)^{6}+A_{4} f_{0}^{\prime \prime}(0)^{8}$

where

$$
\begin{aligned}
A_{0}= & -\left(3928575 \beta^{4}+12315190 \beta^{3}-11915364 \beta^{2}\right. \\
& -59037048 \beta-39690000) \\
A_{1}= & \left(14553000 \beta^{3}-52955280 \beta^{2}-271902960 \beta-222264000\right) \\
A_{2}= & \left(64827000 \beta^{2}+500094000 \beta+555660000\right) \\
A_{3}= & -(555660000 \beta+1111320000)
\end{aligned}
$$

$A_{4}=-277830000+1016064000$

The fixed point is one of the real polynomial roots $\left(\sum_{i=1}^{4} f_{i}^{\prime \prime}(0)=0 \Rightarrow P\left(f_{0}^{\prime \prime}(0)\right)=0\right)$ which certainly shows itself through the recursive process (see Fig. 7). By analysis, 4th order of approximation in each given value of $\beta$ shows solutions in which the maximum deviation (up to $\beta=5$ ), compared to the high-order HAM results, Does Not exceed $0.1 \%$; e.g. for $\beta=5$ it was computed by HAM after handling the controlling parameter and from the 25thorder of approximation that $f^{\prime \prime}(0)=-1.9424$, whist the new technique shows $f^{\prime \prime}(0)=-1.9443$ which is indeed a good match, still below $0.1 \%$ error.

\subsection{Numerical solutions}

Equation 97 is initially expressed as:

$$
\begin{aligned}
& F=f(\eta) \\
& F^{\prime}=Y \\
& Y^{\prime}=Z \\
& \left(1-F^{2} \beta\right) Z^{\prime}+(1+2 \beta Y) F Z-Y^{2}=0 \\
& F(0)=0, \quad Y(0)=1, \quad Z(0)=\alpha
\end{aligned}
$$

Following the instruction given in the previous section, from the 1st-order HPM solution the following scheme with $\left|F^{\prime}\left(\eta_{\infty}\right)\right|=10^{-6}$ is considered:

$\eta_{\infty}=\frac{1}{\sqrt{\beta+1}} \ln \left[\frac{(8+9 \beta)}{8(\beta+1)\left|F^{\prime}\left(\eta_{\infty}\right)\right|}\right]$

5th order Runge-Kutta was employed for discretization purpose. An iterative shooting procedure (to the 5th decimal place) was then pursued.

Table 4 shows a comparison between the methods. It should be also noted that for $\beta=0$, Eq. 97 shows a wellknown closed form solution in the form of $f(\eta)=1-e^{-\eta}$; having No secular terms, a point which provides advantage for HPM in such a nonlinear problem, as well as serving as a suitable initial guess in HAM. Therefore, the nonlinearity seems to be quite simpler that the previous one.

\section{Conclusion}

The aim of the present work was to account the easily-iterative HAM system to be further analyzed for some novel and untapped insights. As a gist, the insight was that the zeroth order term may be potential to contain a certain quantity of the exact solution such as $f^{\prime \prime}(0)$ that is normally sought. 


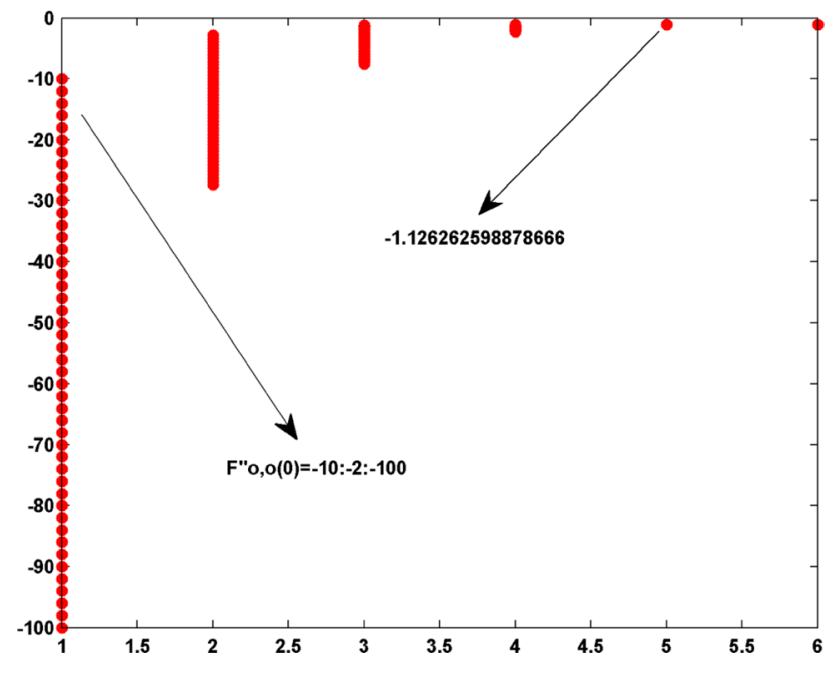

Fig. $7 f_{0, n+1}^{\prime \prime}(0)=T_{4}\left(f_{0, n}^{\prime \prime}(0)\right), n=0,1,2, \ldots,: f_{0, n \rightarrow \infty}^{\prime \prime}(0)=f^{\prime \prime *, 4}(0)=C_{4}$ $\mathrm{X}$-axis is the iteration $(n): \beta=1 / 2$

Through examples we saw that $f_{0}^{\prime \prime}(0)=-\alpha$. The new insight simply states that $f_{0}^{\prime \prime}(0)$ could be $f^{\prime \prime}(0)$ truncated at any homotopy series order; i.e. $f^{\prime \prime, m}(0)$. This hypothesis is checked through Theorem 2 and $f^{\prime \prime}(0)$ is extracted which is regarded as the unique description of the homotopy series solution for $f^{\prime \prime}(0)$ in an order of approximation.

It was shown that unique description of the homotopy series solutions is simply behind a topological feature, the Fixed Point Property, indicating that the homotopy is self-corrector for some certain quantities of the exact solution (here the target quantity was $f^{\prime \prime}(0)$ ). Examples were provided revealing that the present approach is indeed promising.

Acknowledgements The present author would like to dedicate his sincere appreciations to an anonymous reviewer for the constructive comments and suggestions.

Authors' contributions AJ an Independent Researcher, is the sole author of the present work.

\section{Compliance with ethical standards}

Conflict of interests The author declares that there is no competing interest.

\section{References}

1. Liao SJ (1999) An explicit, totally analytic approximate solution for Blasius viscous flow problem. Int J Non-linear Mech 34:759-778

2. Liao SJ (1997) A kind of approximate solution technique which does not depend upon small parameters-II: an application in fluid mechanics. Int J Non-linear Mech 32(5):815-822
3. Liao SJ (1995) An approximate solution technique not depending on small parameters: a special example. Int J Non-linear Mech 30(3):371-380

4. Liao SJ (1992) The proposed homotopy analysis technique for the solution of nonlinear problems. Ph.D. Thesis, Shanghai Jiao Tong University

5. Liao SJ (2003) Beyond perturbation: introduction to homotopy analysis method. Chapman \& Hall/CRC, Boca Raton

6. Liao SJ (2009) Notes on the homotopy analysis method: some definitions and theorems. Commun Nonlinear Sci Numer Simul 14:983-997

7. Liao S-J (2009) A general approach to get series solution of nonsimilarity boundary-layer flows. Commun Nonlinear Sci Numer Simul 14:2144-2159

8. Liao S (2005) Comparison between the homotopy analysis method and homotopy perturbation method. Appl Math Comput 169:1186-1194. https://doi.org/10.1016/j.amc.2004.10.058

9. He JH (2000) A review on some new recently developed nonlinear analytical techniques. Int J Nonlinear Sci Numer Simul 1(1):51-70

10. He J-H (2000) A coupling method of homotopy technique and perturbation technique for nonlinear problems. Int J Nonlinear Mech 35(1):37-43

11. He J-H (1999) Homotopy perturbation technique. Comput Methods Appl Mech Eng 178(3/4):257-262

12. He JH (2006) Homotopy perturbation method for solving boundary value problems. Phys Lett A 350:87-88

13. He J-H (2004) Comparison of homotopy perturbation method and homotopy analysis method. Appl Math Comput 156:527-539

14. He JH (2009) An elementary introduction to the homotopy perturbation method. Comput Math Appl 57:410-412

15. He JH (2005) Periodic solutions and bifurcations of delay-differential equations. Phys Lett A 347:228-230

16. Ariel PD (2009) Extended homotopy perturbation method and computation of flow past a stretching sheet. Comput Math Appl 58:2402-2409

17. Ariel PD (2009) The homotopy perturbation method and analytical solution of the problem of flow past a rotating disk. Comput Math Appl 58:2504-2513

18. Raftari B, Yildirim A (2011) A new modified homotopy perturbation method with two free auxiliary parameters for solving MHD viscous flow due to a shrinking sheet. Eng Comput 28(5):528-539

19. Wang S-Q, He J-H (2008) Nonlinear oscillator with discontinuity by parameter-expansion method. Chaos Soliton Fract 35:688-691

20. Shou DH et al (2007) Application of parameter-expanding method to strongly nonlinear oscillators. Int J Non-linear Sci Numer Simul 8:113-116

21. Jafarimoghaddam A (2018) Two-phase modeling of threedimensional MHD porous flow of Upper-Convected Maxwell (UCM) nanofluids due to a bidirectional stretching surface: homotopy perturbation method and highly nonlinear system of coupled equations. Eng Sci Technol Int J 21:714-726

22. Banach S (1922) Sur Les Operations Dans Les Ensembles Abstraits et Leur Application Aux Equations Integrales. Fund Math 3:133-181 (French)

23. Branciari A (2002) A fixed point theorem for mapping satisfying a general contractive condition of integral type. Int J Math Math Sci 29(9):531-536

24. Kannan R (1968) Some results on fixed points. Bull Calcutta Math Soc 60:71-76

25. Goebel K, Kirk WA (1990) Topics in metric fixed point theory. Combridge University Press, New York 
26. Kutukcu S, Sharma S (2007) A fixed point theorem in fuzzy metric spaces. Int J Math Anal 1(18):861-872

27. Smart DR (1974) Fixed point theorems. Cambridge University Press, London

28. Moradi Sirous, Beiranvand Arezoo (2010) Fixed point of TFcontractive single-valued mappings. Iran J Math Sci Inform $5(2): 25-32$

29. Sajid M, Hayat T, Asghar S (2006) On the analytic solution of the steady flow of a fourth grade fluid. Phys Lett A 355:18-26

30. Liu I-C, Wang H-H, Peng Y-F (2013) Flow and heat transfer for three-dimensional flow over an exponentially stretching surface. Chem Eng Commun 200:253-268

31. Nadeem S, Haq RU, Khan ZH (2014) Heat transfer analysis of water-based nanofluid over an exponentially stretching sheet. Alex Eng J 53:219-224

32. Ashraf MB, Alsaedi A, Hayat T, Shehzad SA (2017) Convective heat and mass transfer in three-dimensional mixed convection flow of viscoelastic fluid in presence of chemical reaction and heat source/sink. Comput Math Math Phys 57(6):1066-1079

33. Ahmad R, Mustafa M, Hayat T, Alsaedi A (2016) Numerical study of MHD nanofluid flow and heat transfer past a bidirectional exponentially stretching sheet. J Magn Magn Mater 407:69-74. https://doi.org/10.1016/j.jmmm.2016.01.038

34. Hayat T, Shehzad SA, Alsaedi A (2016) MHD three-dimensional flow by an exponentially stretching surface with convective boundary condition. J Aerosp Eng 27:04014011. https://doi. org/10.1061/(ASCE)AS.1943-5525.0000360

35. Khan JA, Mustafa M, Hayat T, Sheikholeslami M, Alsaedi A (2015) Three-dimensional flow of nanofluid induced by an exponentially stretching sheet: an application to solar energy. PLoS ONE 10(3):e0116603. https://doi.org/10.1371/journal.pone.0116603

36. Jafarimoghaddam A (2019) On the homotopy analysis method (HAM) and homotopy perturbation method (HPM) for a nonlinearly stretching sheet flow of Eyring-Powell fluids. Eng Sci Technol Int J 22:439-451

37. Mabood F, Khan WA, Ismail AIMd (2017) MHD flow over exponential radiating stretching sheet using homotopy analysis method. J King Saud Univ Eng Sci 29:68-74

38. Hamoud AA, Ghadle KP (2018) Homotopy analysis method for the first order fuzzy Volterra-Fredholm integro-differential equations, Indonesian J. Elec Eng Comp Sci 11(3):857-867
39. Hamoud AA, Ghadle KP (2018) Usage of the homotopy analysis method for solving fractional Volterra-Fredholm integrodifferential equation of the second kind. Tamkang J Math 49(4):301-315

40. Hamoud AA, Azeez AD, Ghadle KP (2018) A study of some iterative methods for solving fuzzy Volterra-Fredholm integral equations, Indonesian J. Elec Eng Comp Sci 11(3):1228-1235

41. Jafarimoghaddam $A$, Aberoumand $H$, Aberoumand $S$, Arani AAA, Habibollahzade A (2017) MHD wedge flow of nanofluids with an analytic solution to an especial case by Lambert W-function and homotopy perturbation method. Eng Sci Technol Int J 20:1515-1530

42. Lighthill MJ (1949) A technique for rendering approximate solutions to physical problems uniformly. Philos Mag 40:1179-1201

43. Awais M, Hayat T, Ali A (2016) 3-D Maxwell fluid flow over an exponentially stretching surface using 3-stage Lobatto IIIA formula. AIP Adv 6:055121

44. Hayat Tasawar, Aziz Arsalan, Muhammad Taseer, Alsaedi Ahmed (2017) Three-dimensional flow of nanofluid with heat and mass flux boundary conditions. Chin J Phys 55:1495-1510

45. Hayat T, Abbas Z, Sajid M (2006) Series solution for the upperconvected Maxwell fluid over a porous stretching plate. Phys Lett A 358:396-403

46. Abel MS, Tawade JV, Nandeppanavar MM (2012) MHD flow and heat transfer for the upper-convected Maxwell fluid over a stretching sheet. Meccanica 47:385-393. https://doi. org/10.1007/s11012-011-9448-7

47. Ishak N, Hashim H, Mohamed MKA, Sarif NM, Khaled M, Rosli $\mathrm{N}$, Salleh MZ (2015) MHD flow and heat transfer for the upper convected Maxwell fluid over a stretching/shrinking sheet with prescribed heat flux. AIP Conf Proc 1691:040011. https://doi. org/10.1063/1.4937061

48. Halim NA, Noor NFM (2015) Analytical solution for Maxwell nanofluid boundary layer flow over a stretching surface. AIP Conf Proc 1682:020006. https://doi.org/10.1063/1.4932415

Publisher's Note Springer Nature remains neutral with regard to jurisdictional claims in published maps and institutional affiliations. 